\title{
Images symboliques du corps et du sport en éducation physique et paradigmes sociologiques
}

Yves Le Pogam

\section{(2) OpenEdition}

1 Journals

Édition électronique

URL : http://journals.openedition.org/trema/2125

DOI : 10.4000/trema.2125

ISSN : 2107-0997

Éditeur

Faculté d'Éducation de l'université de Montpellier

\section{Édition imprimée}

Date de publication : 1 décembre 1995

Pagination : 35-50

ISSN : 1167-315X

\section{Référence électronique}

Yves Le Pogam, «Images symboliques du corps et du sport en éducation physique et paradigmes sociologiques », Tréma [En ligne], 8 | 1995, mis en ligne le 20 septembre 2013, consulté le 19 avril 2019. URL : http://journals.openedition.org/trema/2125 ; DOI : 10.4000/trema.2125

Ce document a été généré automatiquement le 19 avril 2019

Trema 


\title{
Images symboliques du corps et $d u$ sport en éducation physique et paradigmes sociologiques
}

\author{
Yves Le Pogam
}

1 Les rapports entre le sport et cette discipline d'enseignement qu'est l'Éducation Physique demeurent conflictuels puisque les images sur lesquelles cette discipline construit son identité se réfèrent à un ensemble de pratiques extérieures connotées socialement et culturellement, la fragilisant dans sa recherche d'une autonomie. Mais c'est bien le corps qui est l'enjeu de ces malentendus provoqués par les images floues générées par ces proximités, parce qu'il est la donnée première à prendre en compte dès qu'il s'agit d'Éducation Physique. Curieusement d'ailleurs, cette notion de corps est exclue d'une définition possible de l'Éducation Physique qui pourtant est bien une discipline exerçant une action normative à son égard dont les valeurs dépendent d'un contexte culturel, idéologique et politique. Toute approche du corps implique des enjeux métaphysiques et philosophiques qui divisent inévitablement les acteurs du champ.

2 Devant ces turbulences liées à une impossible définition consensuelle de l'Éducation Physique - puisque le corps peut en être absent - ainsi qu'à des théories ou des méthodes incapables heureusement d'en fixer un sens définitif et clos et de triompher d'une vérité, la tentation est forte pour le sport de se substituer à elle. Ne représente-t-il pas en effet une pratique moins contestable a priori qui apporte immédiatement des valeurs facilement repérables comme la santé, le plaisir, des qualités morales recherchées pour une socialisation efficace, c'est-à-dire un ensemble de valeurs qui le justifient comme composante de l'éducation? L'Éducation Physique peut alors s'approprier le sport sans crainte mais en perdant son autonomie puisque les fins et les modalités corporelles se décident ailleurs et son identité se trouble parce qu'elle se confond avec celle du sport. Cette surimpression d'images brouille la clarté de l'identité de l'Éducation Physique car la réalisation d'autres possibles corporels se trouve relativisée face à la surpuissance des techniques sportives façonnant le corps selon des modèles établis malgré la variabilité de 
leurs formes, bornant ainsi d'autres modalités expressives corporelles qui pourraient apparaitre cependant comme autant de manières louables d'accomplissement du corps. Il devient nécessaire de discuter l'idée de l'autonomie de l'Éducation Physique par rapport au sport qui l'a envahi. Comment l'Éducation Physique peut-elle revendiquer son autonomie en affirmant sa volonté de se gouverner selon ses propres lois? Comment peut-elle conquérir sa souveraineté, affirmer sa liberté et son souci d'indépendance si ce n'est en se dégageant de l'emprise et de l'obéissance servile aux règles d'une autorité quelconque ? Est-il réaliste de poser l'idée d'une autonomie de l'Éducation Physique qui contribuerait à la clarification de son image et qui l'érigerait en discipline souveraine ?

3 La sociologie, ou plutôt les sociologies sont attentives à comprendre le problème de l'autonomie en élaborant des théories diverses qui ne sont jamais détachées d'une volonté concrète d'application sur le terrain. En cela elles contribuent à des orientations divergentes de l'Éducation Physique en construisant des images du corps jamais neutres, ainsi que les pratiques corporelles qui leur correspondent. Les images générées par ces théories sur le corps en Éducation Physique et sur le sport sont alors des images symboliques, au sens où elles signifient bien plus qu'elles ne le prétendent : au-delà de leur contenu objectif saisissable, elles montrent une idée ou une chose à travers une autre de telle sorte qu'elles ne peuvent se réduire au seul référent. Elles actualisent un sens latent. Leur aspect figuratif, c'est-à-dire les représentations qu'elles offrent du corps est symbolique au sens où elles ne sont pas des images-signes dont le contenu équivaudrait à ce qui est représenté, mais elles expriment un sens latent dont les interprétations sont ambivalentes étant donné les références divergentes prêtées au symbole.

Il est possible d'admettre que la variété de ces images symboliques agit sur l'imaginaire des acteurs en provoquant en eux des désaccords sur le sens à attribuer aux pratiques corporelles présentes en Éducation Physique, images qu'il est nécessaire de comparer dans la mesure où elles créent des représentations différentes du rapport corps-sportÉducation Physique. Toutes les théories sociologiques ne seront pas convoquées ici. Leur choix repose sur le fait qu'elles font référence à la notion d'autonomie, qu'elles excèdent le seul rapport corps-sport-institution scolaire au sens où elles ont une portée générale permettant de comprendre la réalité sociale, qu'elles bénéficient d'une autorité incontestée dans le champ intellectuel et surtout qu'elles se construisent sur des paradigmes différents générant des images symboliques différenciées. Cette notion de paradigme n'est pas définie ici au sens de Thomas S. Kuhn, c'est-à-dire un accord ou un ensemble de convictions partagé par la communauté scientifique mondiale ${ }^{1}$, mais s'entend par le fait qu'une théorie privilégie un certain nombre de relations logiques au détriment d'autres, de sorte qu'un paradigme "contrôle la logique d'un discours. Le paradigme est une façon de contrôler à la fois la logique et la sémantique $»^{2}$. Alors il devient important pour notre objet de voir comment des paradigmes différents travaillent à la fabrication d'images dont les effets viseront aussi à transformer les pratiques corporelles de manière concrète, mais de façon divergente. Pour cela, le projet de Jean-Michel Berthelot sera suivi momentanément ici, projet qui vise en dépassionnant les débats, à interroger la connaissance du social en montrant les matrices de relations, c'est-à-dire les schèmes qui fondent les modes d'intelligibilité pluriels pris dans des théories anthropo-sociales, ceci en cherchant leur enracinement logique et symbolique ${ }^{3}$. Trois théories qu'il est possible de distinguer par des schèmes d'intelligibilité différents sont retenues ici : le schème dialectique, lisible dans la pensée marxiste qui sera illustré pour notre objet par la critique que Jean-Marie Brohm adresse au sport et à ses effets 
produits au niveau de l'image du corps en Éducation Physique, le schème structural tel qu'il peut être appréhendé dans les théories de Pierre Bourdieu et le schème fonctionnel tel qu'il apparaît dans la pensée systémique saisie dans la particularité des théories d'Edgar Morin. Ces trois matrices d'opérations ou paradigmes qui engendrent une intelligibilité différente du social n'ont pas la même autorité dans le champ de l'Éducation Physique et du sport et s'y inscrivent dans des moments différents. Les travaux de JeanMarie Brohm précèdent dans le champ ceux de Pierre Bourdieu et la pensée d'Edgar Morin commence seulement à engendrer des perspectives de recherche d'un point de vue sociologique. De même la réception de ces trois paradigmes diffère dans notre champ. Si les analyses du sport de Jean-Marie Brohm se connotent fortement d'une image politique, celles de Bourdieu sur le sport, pourtant attachées aussi à une critique de la domination culturelle, sont reçues différemment.

Ce type d'entrée participe d'une volonté d'analyser les différences de conceptions mises en jeu dans la connaissance du social et appelle à repérer les visions opposées de la société qu'elles engendrent de telle sorte que les débats se déroulent sur des querelles qui doivent être clarifiées afin d'en saisir les significations et les enjeux. Ici se retrouve aussi le projet de Pierre Ansart, celui de montrer les enjeux sociaux portés par les différents paradigmes et leurs rivalités dont les conséquences ne sont pas seulement intellectuelles mais profondément concrètes si bien que les paradigmes ne sont jamais sans rapport avec les enjeux politiques: «à la querelle des paradigmes participe donc, dans une certaine mesure, le heurt des idéologies politiques, avec le jeu subtil des non-dits et des proclamations, non sans contradictions et dénégations $»^{4}$.

Saut sur place avec élévation simultanée des jambes tendues.

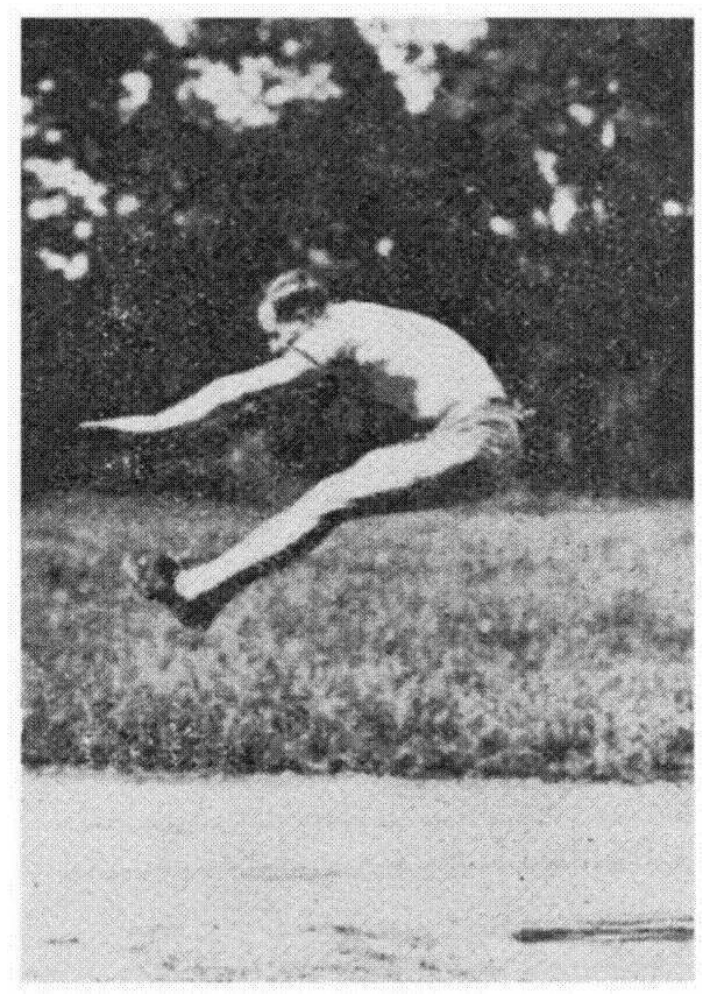

Sous secrétariat d'état de l'éducation physique, Règlement Général d'Éducation Physique, Méthode Française, Deuxième Partie, Paris, Charles Lavauzelle et Compagnie, 1933. 
6 Après avoir dégagé les schèmes dominants des trois théories sociologiques retenues et les images symboliques du corps et du sport qu'elles construisent, il sera nécessaire d'en montrer les enjeux concrets et symboliques par l'analyse de leurs polémiques ainsi que les effets qu'elles produisent relativement au corps en Éducation Physique. Elles participent en tout cas à la dynamique identitaire de l'Éducation Physique, par les idées émises, par la création ou par le renouvellement des images symboliques qu'elles provoquent et par leurs effets sur l'imaginaire des acteurs impliqués dans le procès éducatif.

\section{Schème dialectique et images symboliques du corps et du sport}

7 Jean-Michel Berthelot propose une typologie des schèmes d'intelligibilité en l'illustrant par l'analyse de textes de référence clairement dominés par un schème et pouvant remplir une fonction de paradigme en sociologie. L'auteur s'appuie sur un extrait du Capital pour faire émerger ce schème dialectique qu'une tradition philosophique illustre par l'idée de contradiction. Dans cette perspective, un phénomène est saisi comme la résultante d'un système contradictoire défini par l'existence de deux termes à la fois indissociables et opposés, chaque terme étant impliqué par l'autre et entrant en contradiction avec lui.

8 C'est bien une sociologie dialectique à laquelle s'attache Jean-Marie Brohm, dans un procès de démystification de ce que la logique sportive tend à occulter, c'est-à-dire l'ensemble des contradictions qui lui sont contingentes ${ }^{5}$. En se référant aux Théories de la plus-value dans lesquelles Marx note comment l'apologie de la production n'aboutit jamais à la crise en falsifiant les faits économiques afin d'affirmer une unité face à une contradiction qui éclate cependant lors d'une catastrophe dont l'analyse n'est jamais faite, Jean-Marie Brohm transfère analogiquement cette thèse au sport où les idéologues nient aussi l'objectivité de la contradiction qu'ils présentent comme des dysfonctionnements passagers (qu'il s'agisse de violence, de l'apolitisme, de l'idéologie pacifiste et humaniste) alors que la crise est constitutive des structures mêmes du sport ${ }^{6}$. Cette crise provient de la combinaison dialectique de trois données: "d'une part les contradictions du système social capitaliste qui se sont réfractées de manière aiguë dans l'institution sportive; d'autre part les contradictions internes spécifiques de ladite institution qui se sont combinées aux contradictions sociales externes; et enfin les contradictions nées des contestations incessantes provenant des milieux intellectuels critiques et même de certains sportifs progressistes ${ }^{7}$. Révéler donc la condensation dans le sport des structures du capitalisme devient une tâche urgente car « le destin sportif est un destin capitaliste $»^{8}$ bien éloigné de l'isolement humaniste dans lequel il se confine, et le procès dialectique doit s'accompagner de l'analyse du principe de rendement constitutif du fonctionnement de la société capitaliste et du sport sous la forme du rendement corporel. Le sport, superstructure idéologique, ne doit pas être réduit à l'idée de « reflet » par homologie au système capitaliste, car il s'agit d'un élément participant à son édification. Ainsi les catégories centrales du sport (record, mesure, compétition, rendement), ne sont pas «le simple reflet mécanique du capitalisme, mais les matrices mêmes de toutes les institutions reposant sur l'économie marchande capitaliste, laquelle ne peut 
pas se comprendre sans les catégories de temps abstrait, d'argent, de capital, de marchandise, de plus-value, etc., $»^{9}$.

Ce qui se pose ici par rapport à notre objet, c'est l'idée de l'autonomie de cette superstructure idéologique qu'est le sport et les relations qu'il entretient avec l'infrastructure, ceci dans le cadre du schème dialectique. Dans la logique de Jean-Marie Brohm, il ne saurait y avoir dans ce sens d'autonomie structurelle du sport dans sa liaison avec la société capitaliste et il s'appuie en cela sur les thèses de Gramsci et celles d'Althusser qui ont en commun de privilégier leur rapport dialectique. En effet Gramsci ne cesse de montrer que ces deux moments sont essentiels au devenir historique d'un phénomène, chacun étant en même temps le moteur et le frein de l'autre ${ }^{10}$. Par la notion d'Appareils Idéologiques d'État (A.I.E.), Louis Althusser entend aussi signifier le rôle de l'existence matérielle (la fonction pratico-sociale) de l'idéologie clans les institutions et les pratiques en ne la considérant pas seulement- comme c'était le cas dans la tradition marxiste- comme une forme privilégiant le problème de la connaissance En réaction à un déterminisme économiste, il montre la puissance des superstructures et invite à repérer leurs fonctions sociales et leurs liens- comme Gramsci l'avait entrepris avant lui- avec les intérêts de l'État ou des classes et les structures psychologiques des sujets. En ce sens, l'école, la famille, la religion, la culture (dans laquelle outre les Lettres et les Beaux-Arts, Althusser inclut le sport), sont des A.I.E. au sein desquels existe une lutte de classes " puisque aucune classe ne peut durablement détenir le pouvoir d'État sans exercer en même temps son hégémonie sur et dans les A.I.E. $»^{11}$. Au-delà de leur diversité, une unité existe entre les A.I.E. du fait qu'ils réalisent l'idéologie dominante d'une classe, et on comprend que l'idéologie ait une existence matérielle, une réalité opérationnelle par son lien aux classes et à l'État. Les institutions et les appareils deviennent leurs lieux d'expression avec leurs pratiques et leurs rituels dont l'effet sur les normes et les goûts est patent au niveau des comportements de la vie quotidienne. L'unité entre la superstructure et l'infrastructure n'est pas mécanique mais dialectique et la métaphore gramscienne de « ciment » traduit bien l'idée de cohésion, d'ajustement pour marquer la diffusion de l'idéologie. Pour Jean-Marie Brohm le sport est un A.I.E., qui en s'intégrant à l'appareil d'État, s'étend à toute la sphère quotidienne en diffusant l'idéologie dominante. La liaison État-politique-sport est alors évidente et dans le sport, lié à l'appareil culturel, circule l'idéologie dominante, comme protection de la domination d'une classe ${ }^{12}$. L'objectif de cette sociologie dialectique est de révéler cette logique de la domination en démystifiant l'idéologie qui occulte la structure réelle des rapports de production naturalisés et en montrant que «L'idéologie sportive, comme toutes les idéologies, contribue par conséquent à la reproduction élargie des rapports de production ${ }^{13}$.

Le projet d'une telle analyse vise l'émancipation des sciences de l'homme, par la clarification des logiques inconscientes qui nous gouvernent, par le dévoilement de ce que nous croyons nôtre et qui est autre. Ce projet est proche de celui de l'École de Francfort dont le procès de la domination fait jaillir les processus contradictoires visant à une pluralité critique amenant à une société émancipée « qui renoncerait au principe du renoncement $»^{14 .}$

11 Cette théorie de la déconstruction se prolonge naturellement par une critique du corps dans l'institution scolaire puisqu'il n'est pas possible d'autonomiser l'appareil scolaire profondément dépendant des autres appareils et de l'État de classe. Il serait donc illusoire de penser que l'école ne promeuve pas ses intérêts, rompant ainsi avec le mythe du partage de la culture. Le corps se lie à l'idéologie et il n'est pas étonnant que le sport soit 
valorisé massivement. Les images du sport conduisent à des images symboliques du corps et à des pratiques corporelles montrées comme une arme politique participant du procès critique de la politique étatique et de l'économie dominante ${ }^{15}$. Alors s'affirme encore le projet émancipatoire, la recherche du principe de souveraineté contre les emprises politique, sociale, idéologique, notamment au niveau des institutions, ces «machines à influencer » qui savent que l'essentiel de la persuasion ne passe pas par une action sur la conscience mais sur le corps ${ }^{16}$.

Dans ce sens, l'image du corps en Éducation Physique se fonde sur l'idéologique et il n'existe pas de neutralité axiologique d'un point de vue institutionnel. Les finalités dépendent d'un État de classe ${ }^{17}$ et l'important est alors de "dévoiler les mécanismes de légitimation du discours éducatif, donc de son pouvoir et corrélativement de la croyance qu'il suscite $»^{18}$. Il s'agit encore de démythifier le pouvoir du sport sur le corps de l'enfant qui le réduit à un être « apprenant ", à « un agent d'exécution de programmes moteurs ", occultant la prise en compte de la globalité du sujet désirant et agissant, de ses projets, de ses intentionnalités et de ses finalités ${ }^{19}$. La logique de domination que le sport exerce sur le corps de l'enfant est de nouveau révélée (importance du travail, du réel, des apprentissages, du commandement, du rendement), contre la « poétique » de l'action ${ }^{20}$.

Suspension s et appuis, échelles jumelles, exercices les pieds sur le sol.

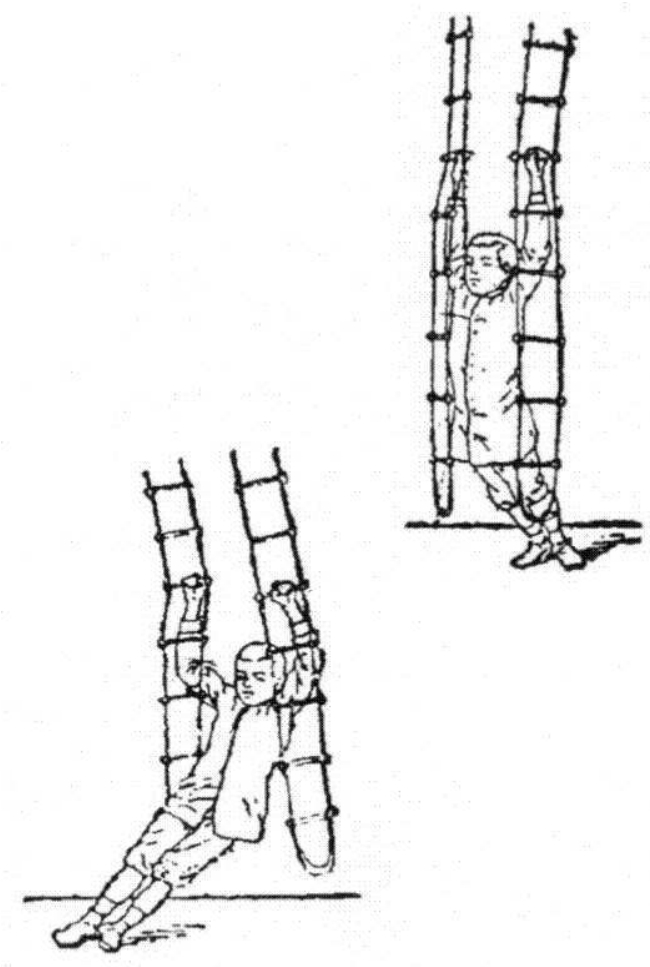

Ministère de l'Instruction Publique, Manuel d'Exercices Physiques et de Jeux Scolaires, Paris, Hachette, 1 réed., 1908.

On l'aura compris, armé de théories multi-référentielles, le projet est ici délibérément émancipatoire pour le sujet et ne peut s'articuler en Éducation Physique que sur des pratiques corporelles permettant la liberté du sujet ce qui réfute toute clôture corporelle portée par les structures sportives compétitives analogues à celles que véhicule l'idéologie dominante. L'institution scolaire ne peut produire un corps souverain 
puisqu'elle n'est pas autonome. Conduire le procès de cette absence d'autonomie constitue la tâche de la sociologie dialectique, ou de toute sociologie critique d'ailleurs, dans la mesure où en objectivant les mécanismes qui contribuent à notre aliénation, elle engage, par une conscientisation, un projet émancipatoire de libération.

\section{Schème structural et images symboliques du corps et du sport}

14 Le texte choisi par Jean-Michel Berthelot permettant de révéler le schème structural est extrait de Anthropologie structurale de Claude Lévi-Strauss ${ }^{21}$. Pierre Bourdieu construit son "structuralisme génétique » en rompant avec le structuralisme de la phonologie de Claude Levi-Strauss trop mécaniste et avec celui de Louis Althusser qui considère les agents comme le reflet de la seule incorporation des structures. Pierre Bourdieu insiste au contraire sur la dimension des affects des agents qui ne sont pas le simple reflet de structures objectives, même s'ils dépendent d'elles ${ }^{22}$.

En tenant compte de ce schème d'intelligibilité, comment parvenir à dégager une cohérence dans la théorie de Pierre Bourdieu amenant à mieux comprendre l'idée d'autonomie et à la rapporter aux images du corps et du sport dans la perspective d'en montrer les incidences dans l'institution scolaire, et plus particulièrement en Éducation Physique ? Répondre à cette question implique que soient clarifiées, sommairement tant elles sont divulguées, les notions de champ et d'autonomie relative, notions auxquelles s'attache celle de production symbolique.

L'idée dominante qui rompt ici avec les problématiques marxistes est le refus de la dichotomie infrastructure-superstructure car même si leur rapport se perçoit de façon dialectique comme il a été vu, cela suppose toujours malgré tout que l'économie triomphe en dernier lieu. Ici, le schème structural vise à dévoiler les relations et quel système de relations compose un champ précis, qu'il s'agisse du sport, de la science, de la mode, etc. Ces champs sont composés d'agents régis par un système de positions incluant des relations jamais dégagées d'une subjectivité, même si celle-ci est profondément dépendante des structures qui organisent les visions du monde, les jugements ou les comportements des agents qui les composent. Théorisée dans le texte Champ intellectuel et projet créateur $^{23}$, la notion de champ est immédiatement liée à celle d'autonomie relative et va ensuite s'étendre à d'autres objets. L'auteur entendait montrer à propos de la création intellectuelle que le champ « est irréductible à un simple agrégat d'agents isolés, à un ensemble additif d'éléments simplement juxtaposés » mais - à la façon d'un champ magnétique, (il) constitue un système de lignes de force : c'est-à-dire que les agents qui en font partie peuvent être décrits comme autant de forces qui, en se posant, s'opposant et se composant, lui confèrent sa structure spécifique à un moment donné du temps $»^{24}$. L'idée de champ s'articulait déjà avec une nécessité, celle de le considérer dans une autonomie relative, "autorisant l'autonomisation méthodologique qu'opère la méthode structurale en traitant le champ intellectuel comme un système régi par ses lois propres ", ce champ est produit par une histoire et l'état d'un champ ne tient pas à des "vérités d'essence, transhistoriques et transculturelles $»^{25}$. Cette autonomie du champ permet de comprendre comment historiquement il se construit progressivement en s'affranchissant de l'économie ou du social, de telle sorte que la valeur esthétique d'une œuvre reste irréductible à la valeur économique. Enfin dans ce texte majeur émerge l'idée de la 
légitimité culturelle, puisque dans toute société existe des «puissances sociales » (hautes classes, institutions scolaires, critiques littéraires etc.,) qui en raison de leur « pouvoir économique et politique ou de garanties institutionnelles dont elles disposent, sont en mesure d'imposer des nonnes culturelles à une fraction plus ou moins étendue du champ intellectuel » si bien qu'une hiérarchie s'instaure selon le degré de légitimité qu'elles imposent aux pratiques culturelles, c'est-à-dire selon un système de règles permettant de les qualifier selon leur degré de dignité.

Cette théorisation de l'autonomie relative s'appliquera aussi au champ sportif, «il s'ensuit que l'on ne peut pas comprendre directement ce que sont les phénomènes sportifs à un moment donné dans un environnement social donné en les mettant en relation directement avec les conditions économiques et sociales des sociétés correspondantes : l'histoire du sport est une histoire relativement autonome qui, dès lors qu'elle est scandée par les grands événements de l'histoire économique et politique, a son propre tempo, ses propres lois d'évolution, ses propres crises, bref, sa chronologie spécifique $\aleph^{26}$. Pour cela, seront convoqués les puissances sociales légitimantes permettant de comprendre la genèse relativement autonome de la production et de la circulation des produits sportifs, comme le rôle des grandes écoles, les règlements universels permettant les échanges entre les institutions, l'affirmation d'une définition bourgeoise de l'éducation par le sport (énergie, courage, esprit d'entreprise) contre la définition de la petite bourgeoisie traditionnelle (savoir, érudition, docilité scolaire), si bien que la définition du sport est un enjeu de lutte de concurrence dans le champ de production symbolique dont $l$ 'enjeu vise à la légitimation de ce « bien symbolique » qu'est le sport et qui tout en ayant une valeur marchande vaut aussi par sa signification que lui confère une autorité à l'intérieur du champ en établissant des distinctions pertinentes. Au nom de cette légitimité cul-airelle, tous les sports ne sont pas dignes de faire l'objet du discours distingué et les différenciations sont $l$ 'objet d'une lutte pour le classement puisque les choix reposent sur des préférences, sur des goûts dépendants de la structure du champ et des positions qu'y occupent les classes. 
Mains aux hanches. Élévation avant et écartement intégral du genou.

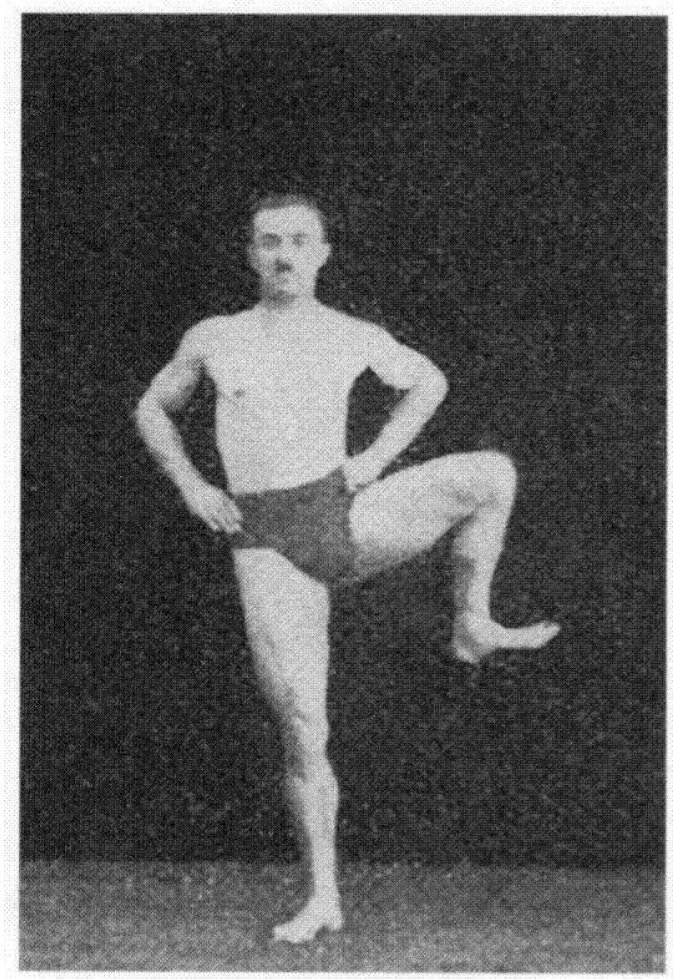

Sous secrétariat d'état de l'éducation physique, Règlement Général d'Éducation Physique, Méthode Française, Deuxième Partie, Paris, Charles Lavauzelle et Compagnie, 1933.

Mais ce qui transparaît de cette théorisation c'est la logique de domination, une dimension politique commune àla sociologie de Jean-Marie Brohm, trop souvent occultée dans le champ des STAPS dans lequel pourtant les théories de Pierre Bourdieu font autorité. Tout se passant comme si la notion de violence symbolique était neutralisée dans l'analyse de la dimension culturelle des pratiques. Or cette notion permet de comprendre le passage qui s'effectue de la sociologie du sport àl'institution scolaire et la place et le rôle qu'elle accorde au corps. Le problème ne concerne plus seulement les enjeux de légitimation du corps en Éducation Physique fondés sur une définition bourgeoise de la culture sportive, mais bien ce que cette légitimation impose aux autres classes qui en subissent la définition. Cette question est curieusement occultée dans les STAPS alors qu'elle constitue une composante essentielle dans l'œuvrede Pierre Bourdieu qui depuis Les Héritiers et La Reproduction ne cesse de montrer comment l'institution scolaire travaille àla transmission d'une culture légitime ${ }^{27}$. Certes relativement àla question précise du corps en Éducation Physique, Pierre Bourdieu ne se livre àaucune analyse, mais ilest possible de tenter quelques pistes àpartir de ses critiques de l'institution. En ce sens, l'école n'a plus une fonction d'endoctrinement, mais exerce plutôt une fonction de légitimation de l'ordre établi, une fonction de conservation de la structure de classe. C'est làune manière de voir comment l'école, au nom de son autonomie relative, traite de la culture populaire et des valeurs corporelles qui la caractérisent, car l'école inculque non pas des opinions ou des attitudes mais des schèmes inconscients àpartir desquels s'organisent pensée et action (les habitus) en imposant donc un arbitraire culturel. Cette violence symbolique réalisée par le travail pédagogique est un substitut de la contrainte physique. Mais on comprend mieux la place que pourrait 
attribuer Pierre Bourdieu àl'Éducation Physique, quand d'un point de vue anthropologique, ils'attache àmontrer l'incorporation des structures dans la prime éducation où le travail pédagogique consiste - àsubstituer au corps sauvage, et en particulier àl'éros $a$-socialqui demande satisfaction àn'importe quel moment sur le champ, un corps "habitué », c'est-à-dire temporellement structuré », et si les institutions attachent un prix « aux détails en apparence les plus insignifiants de la tenue, du maintien, des manières corporelles et verbales, c'est que, traitant le corps comme une mémoire, elles lui confient sous une forme abrégée et pratique, c'est-à-dire mnémotechnique, les principes fondamentaux de l'arbitraire culturel [...]. Toute la ruse de la raison pédagogique réside précisément dans le fait d'extorquer l'essentiel sous l'apparence d'exiger l'insignifiant $»^{28}$.

19 Ici s'exprime toute la différence méthodologique entre les analyses de Jean-Marie Brohm relatives à une sociologie du sport mise en rapport avec l'institution scolaire et celles de Pierre Bourdieu. Le schème dialectique majore les relations entre les différentes instances et les institutions en privilégiant la globalité (conjonction État-économie-idéologieculture-institutions) et en relativisant du même coup (sans la nier) la dimension interne des institutions propres (scolaire ou sportive), tandis que le schème structural maximise au plan méthodologique le pôle de l'autonomie relative d'un champ en partant du postulat qu'il est régulé par des normes propres, neutralisant (sans l'occulter) le poids de l'économie ou du social extérieur au champ. Alors que le caractère inséparable des appareils est prononcé dans le premier cas et que l'absence d'autonomie est reconnue, les relations entre les champs ne sont pas recherchées dans le second cas, sauf dans la recherche d'homologies structurales entre eux mais chaque champ conserve une originalité et une pertinence (le champ sportif et le champ scolaire sont traités méthodologiquement de la même manière, mais les agents, leurs histoires, les violences symboliques, les relations qu'ils connaissent sont spécifiques de chacun d'eux). Les images symboliques du corps et du sport ainsi formées en Éducation Physique ne renvoient ni aux mêmes analyses ni ne convergent vers un même sens, malgré la référence commune des deux auteurs à la logique de domination, à la reconnaissance d'une homologie structurale et à une humeur anti-institutionnelle. Le schème dialectique repéré chez Jean-Marie Brohm invite à une lecture du corps en Éducation Physique médié par le sport et renvoie à la reconnaissance d'une puissance étatique et à l'affirmation du pouvoir d'une classe dans l'objectif de dévoiler les processus d'idéologisation divulgués dans un appareil scolaire qui prétend à la neutralité culturelle sur le corps. Le schème structural lu depuis les théories de Pierre Bourdieu invite aussi à une déconstruction de l'idée de neutralité culturelle des pratiques mais qui est réalisée par une pluralité de puissances en concurrence qui tentent de doter le corps en Éducation Physique, au moyen d'un système de croyances, d'une image légitime ou en voie de légitimation fixant alors un arbitraire culturel conforme à leurs intérêts. On comprend l'hostilité que voue Pierre Bourdieu à la notion d'Appareil et à la notion de complot qu'il lui associe (même s'il reconnaît qu'en certaines circonstances le champ peut se mettre à fonctionner comme un appareil, par exemple dans l'écrasement du dominé par le dominant), le champ étant formé par un ensemble de relations, de "particules" sous l'emprise des affects (attraction, répulsion) jamais tirées mécaniquement et poussées par des forces extérieures, relativisant ainsi le pouvoir marxiste du champ économique et de l'État, cette notion « quasi métaphysique ", « à faire voler en éclat ${ }^{29}$. 


\section{Schème fonctionnel et images symboliques du corps et du sport}

20 Le principe en œuvre clans le schème fonctionnel discriminé par Jean-Michel Berthelot consiste à montrer que la relation entre un élément d'un système et le système lui-même est de type circulaire. Le système n'existe que si cet élément remplit une fonction qui est utile à sa survie, par exemple une institution comme l'école ne peut survivre que si elle remplit une fonction dans un système particulier. Ce schème à ses origines tendait, par les théories fonctionnalistes, à considérer la société comme un tout comparable à un organisme, la société ayant un système de besoins que la fonction des institutions était de satisfaire. Ce fonctionnalisme va favoriser l'élaboration du modèle systémique en sociologie puisque son fondement repose sur le concept de système. Les éléments théoriques actuels qui construisent le systémisme proviennent de la rencontre de deux courants. D'abord, d'une réaction critique de certains biologistes contre le fait que le corps ne peut pas être compris comme un tout cohérent dont chacune des parties remplit une fonction nécessaire à l'ensemble et ils proposent contre cela de privilégier non plus l'étude de ces parties isolées l'une de l'autre mais leurs interactions dynamiques. Ensuite d'un courant cybernétique centré sur les problèmes de la circulation de l'information qui intéressent aussi les physiciens, les mathématiciens, avec les notions d'autorégulation, de rétroaction, de relations entre les systèmes. La conjonction de ces deux directions va créer des pôles communs d'interrogation autour de la notion d'organisation et créer des rencontres épistémologiques générées par cette interdisciplinarité, notamment autour de l'idée d'autonomie. Cette notion sera retenue ici en partant du postulat qu'elle contribue à édifier des images du corps et du sport dans les institutions. Le systémisme est probablement appelé dans le milieu de l'éducation à un grand développement car il se présente comme un outil d'intervention et d'analyse du changement de situations très prisé dans la recherche-action, comme un instrument désidéologisé pour le terrain toujours inquiet de la fausse neutralité des sciences ou de leur impérialisme et comme une méthode orientée vers l'efficacité et la prise de décision. Il a connu une grande extension dans les sciences sociales depuis les années 1970, mais c'est probablement les travaux originaux d'Edgar Morin qui contribuent à en accroître l'audience, par un travail sur les concepts d'auto-organisation, de rétroaction, de récursion, d'ordre, de désordre, etc., permettant de développer la notion de complexité et de substituer à une conception scientifique analytique, une science ouverte à une vision globale, à une pluralité idéologique et méthodologique admettant que le déterminisme puisse se combiner avec le hasard, l'inédit et le désordre.

21 Les théories d'Edgar Morin se construisent sur une exigence épistémologique concernant l'idée d'une science dont la dimension anthropologique est constante, signe que les références au modèle systémique n'appellent pas, contrairement à l'usage que certains font du systémisme, à une mécanisation de la pensée ou à une modélisation mathématique occultant le vide de leur construction théorique ${ }^{30}$. Le modèle proposé est ouvert et prend en compte les significations fondées les expressions d'affects, de telle sorte que ce paradigme sorte la science de sa gangue scientiste, ce qui n'est pas fait dans les neurosciences ou les "sciences computationnelles» quand elles excluent la part d'étrangeté ou la réalité spécifique de l'esprit et des phénomènes humains. Les concepts du systémisme ou des théories de l'information pensés clans la logique d'Edgar Morin ne 
peuvent conduire à l'enfermement de la corporéité. L'humain, les sociétés, s'originent dans de l'auto-organisation et de la complexité qui donnent lieu à l'analyse de processus d'émergence, à la générativité des phénomènes, dans un mélange de déterminismes, de hasard, bref d'autonomie.

On ne trouve pas dans les théorisations de l'autonomie d'Edgar Morin de référence à l'infrastructure/superstructure ou à un marxisme mécaniste niant par exemple la dimension de l'événement, ni de référence à la notion de champ, mais l'approfondissement de notions comme celles d'auto-organisation, ou d'autonomie de l'organisation, chaque système créant ses propres déterminations et ses propres finalités, chaque organisation s'organisant elle-même ${ }^{31}$. Bref, ici s'affirme la présence d'une organisation de l'autonomie. L'originalité de l'approche se situe dans le fait que domine ici l'idée d'une circularité dynamique entre les éléments d'un tout que le principe de "causalité récursive » et la métaphore du tourbillon permettent de mieux comprendre. Chaque moment "est à la fois produit et producteur ", chaque processus récursif est un processus où les produits et les effets sont en même temps causes et producteurs de ce qui les produit. Ceci rompt avec la linéarité cause/effet, produit/producteur puisqu'il y a autoproduction, auto-organisation.

Concernant la spécificité de notre objet, au nom de ces principes, parler de l'école ne peut plus se faire sans l'intégrer dans la société qui la fait. Cette théorisation la distingue de l'autonomie méthodologique du champ de Pierre Bourdieu mais se rapproche de la perspective globalisante de l'analyse de Jean-Marie Brohm tout en la complexifiant au nom de la causalité récursive ${ }^{32}$. Les concepts majeurs ne sont plus ceux de l'idéologie ou de la violence symbolique, mais rien n'interdit leur utilisation dans le cadre de la complexité révélée par Edgar Morin. Simplement, une dimension majeure est ici prise en compte, liée au fait que l'école est un système ouvert à des données économiques et politiques générant de l'imprévu qui se combine aux déterminismes des normes et des valeurs défendues par l'institution dont il importe de montrer les rétroactions sur elle (perceptibles dans les innovations à l'école, dont le sens varie selon les contextes) ${ }^{33}$ ,notamment par les crises qui peuvent naître à cette occasion. 
Sauter à califourchon par la croupe avec élan. Sauter à terre à gauche. Exercices physiques pour élèves de 16 ans et au-dessus.

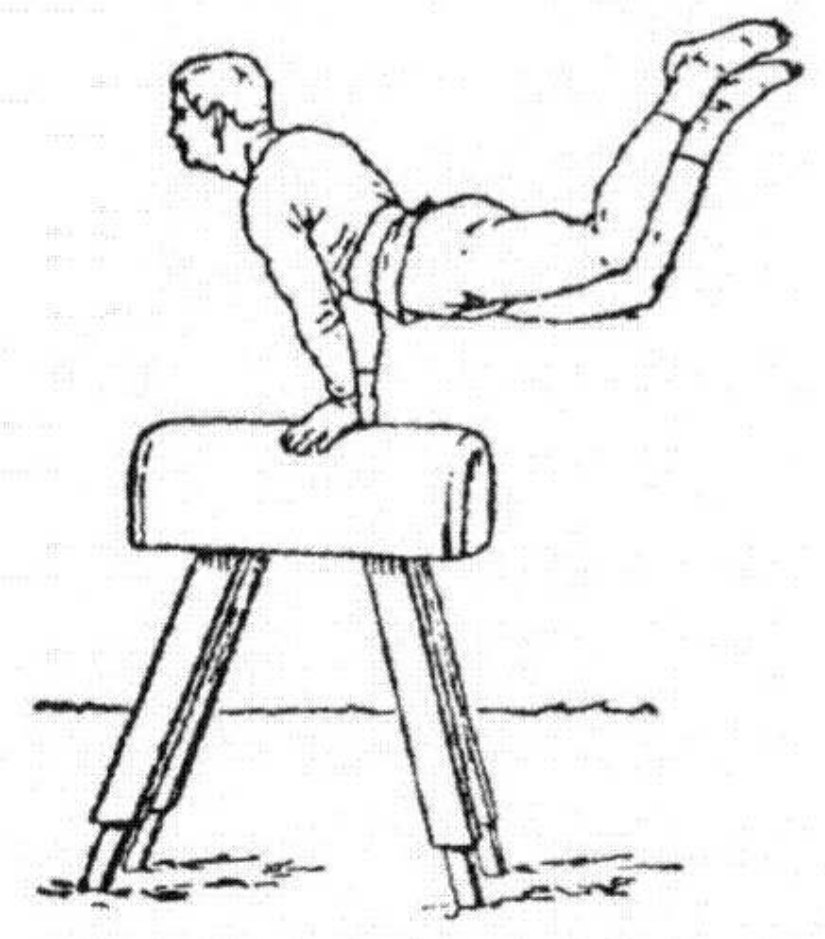

Ministère de l'Instruction Publique, Manuel d'Exercices Physiques et de Jeux Scolaires, Paris, Hachette, 1 réd.,1908.

Quel usage peut-il être fait de cette théorisation au niveau de la construction d'images du corps et du sport et des pratiques corporelles au sein de l'institution scolaire en conservant à l'esprit l'idée de circularité et de récursivité ? Le corps en Éducation Physique ne peut jamais alors être défini une fois pour toutes, circonscrit dans un déterminisme économique ou politique de type causaliste ou pris dans des relations de concurrence entre les agents visant à légitimer une culture physique de classe. L'image dominante qui se dégage est celle d'une labilité générée par la nécessité de se centrer non sur les causes/effets mais sur les désorganisations et réorganisations nées d'imprévus, d'événements ou de hasards. L'éducation corporelle réalisée au sein de l'institution scolaire par l'Éducation Physique est appelée alors à une recomposition incessante intégrant la dualité de l'ordre et du désordre organisateur, n'évacuant pas les contradictions, bref des images symboliques montrant l'incomplétude des vues sur le corps et des pratiques corporelles ainsi que leur production et reproduction incessante sous les injonctions nées de la multiplicité des relations et des rétroactions entre tous les éléments qui composent le système éducatif en prise avec des mutations extérieures qui l'affectent. Les capacités de découverte d'un tel outil dépendent de la qualité et de l'enrichissement que lui accorderont ceux qui travaillent à comprendre la présence d'une générativité dans des institutions soumises aux mutations. Rien ne serait plus réducteur que la transgression du message initial d'ouverture d'Edgar Morin en le métamorphosant en froides analyses de modélisations des comportements oubliant que le corps de l'enfant en Éducation Physique n'est pas assimilable à un montage prédéterminé. Au contraire les images produites conduisent à privilégier l'inattendu, la surprise qui sourdent sous la 
transparence rationaliste des objectifs et des contrats, Il est vrai que la voie de la complexité est difficile à suivre et rompt avec une idée de la recherche très localisée, puisque ici la globalité et l'incertitude prévalent, deux dimensions contradictoires avec l'idéologie dominante des recherches actuelles.

\section{Une réception différenciée} pour cela son caractère mécaniste et son structuralisme froid (le pouvoir hégémonique de l'État et la dépendance des agents face à l'idéologie), tout comme Edgar Morin plaide pour le « retour de l'événement » et le singulier qui font naître l'improbable au niveau de la lutte de classes, aléatoire contenu dans Marx et qui renvoie à des obstacles, à des révolutions qui sont des événements clés qui deviennent générateurs, contrairement à une orthodoxie marxiste "dérivant en dogmatique vulgatique» influencée par un «déterminisme économistique » ou «par la glaciation stalinistique » ou encore «par le structuralisme althussérien ${ }^{34}$.Pourtant il est possible aussi de faire du marxisme une analyse moins finaliste comme en témoignent les fondements du schème dialectique dégagé précédemment. De même il est possible de discuter du rapport entre la science et l'idéologie, idéologie dont est accusé le marxisme alors que la construction de la sociologie s'est faite sur la base idéologique. Pierre Ansart rappelle à ce sujet comment le soupçon d'idéologisation entraîne une épistémologie critique, comme processus de vérification et de déstructuration des schèmes du savoir dominant, bien développés par Saint Simon, Marx ou Proudhon. La pensée politique n'est pas extérieure à leur pensée, elle relie leurs écrits et peut être même positive pour l'auteur qui pose une question renversante, « L'adhésion à une idéologie et la volonté de la promouvoir ne pourrait-elle pas, en certaines situations, constituer l'une des conditions de la clairvoyance et de la connaissance? « Dans ce sens, «L'idéologie politique n'est pas un obstacle à la découverte et à la connaissance mais bien la condition de leur approfondissement ${ }^{35 .} \mathrm{Ce}$ risque idéologique qui soutient la recherche est bien présent aussi dans la sociologie de Pierre Bourdieu, mais sa réception dans le champ est différente et la cause ne tient pas au système de la preuve présent dans les deux types de travaux. Les images du corps et du sport, si elles se construisent sur des schèmes d'intelligibilité dont la légitimité n'est pas sociologiquement contestable puisque la dimension idéologique préalable au questionnement du social est neutralisée par la preuve, leurs effets par contre sont sujets à polémiques, signe que les enjeux de la communauté ne sont pas scientifiques mais sociaux. 

notion de champ et de système et s'ériger contre des ressemblances superficielles. Ici encore, Pierre Bourdieu refuse l'idée fonctionnaliste et organiciste qu'il lit dans les notions d'auto-organisation et d'auto-référentialité de la théorie des systèmes, alors que le systémisme se construit précisément contre cette vue. Mais la critique de Pierre Bourdieu ne vaut que parce qu'il maximise dans ses théories l'idée de lutte et de rapports de force que ne majore pas le systémisme par rapport à la dynamique des champs. Un champ n'a pas de parties ni de composantes, même si Pierre

Bourdieu reconnaît que l'on peut dans les champs «traiter de prises de positions constitutives d'un espace de possibles comme un système $»^{36}$. Le désir de rigueur épistémologique et de neutralité apporté par l'analyse de Jean-Michel Berthelot se complexifie car les théories sont humaines.

L'analyse comparative de trois modes d'appréhension de l'idée d'autonomie à permis de montrer des divergences quant aux images symboliques qu'ils créent. Comprendre le regard qu'ils portaient sur une institution rendait nécessaire ce long détour sur leurs principes fondateurs, tous légitimes mais socialement différenciés dans leur réception. Ces trois modèles, malgré leur différence ont en commun de montrer les dynamismes qui animent l'institution et de plaider pour une raison ouverte, plus critique semble-t-il dans les théories de Jean-Marie Brohm et dans celles de Pierre Bourdieu que dans celles d'Edgar Morin. Certains effets spécifiques au champ des STAPS demeurent tout de même mystérieux quant à la réception. Pourquoi la remise en cause des institutions dont se proclament ouvertement Jean-Marie Brohm et Pierre Bourdieu est-elle mieux acceptée venant du dernier? Pourquoi notre champ n'accepterait pas la critique que Pierre Bourdieu fait des rituels d'institution quand il écrit, «La consécration n'a pas d'autres avantages pour moi que de m'aider à persévérer dans la transgression $»^{37}$ ? Enfin, les STAPS dans leur questionnement sur la corporéité suivront-elles l'ouverture anthropologique d'Edgar Morin ou feront-elles de l'approche systémique une application modélisée sur les procédures cybernétiques ou mathématiques vidées de théorisations sur l'humain? Un devenir pluridimensionnel de l'Éducation Physique qui devra composer avec la force des images chao tiques et contradictoires générant un imaginaire poḯtique dont la dimension finira bien par être légitimée puisqu'elle permet de justifier les valeurs à attribuer au corps, valeurs inconstantes, indéfinies et indéfinissables. 
La canne. Coup de flanc tierce et sa parade.

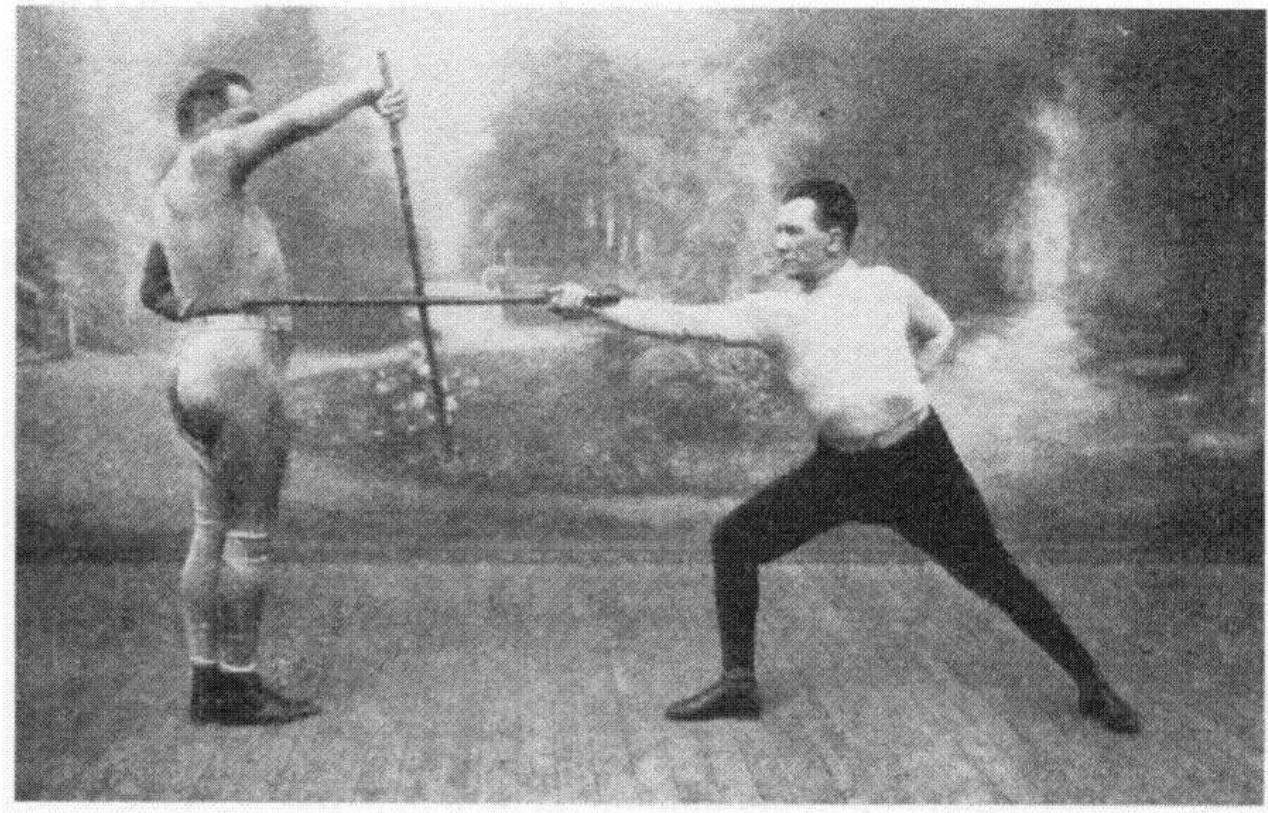

Académie des Sports et Comité National des Sports, Encyclopédie des Sports, Tome I, Paris, Librairie de France, 1924

\section{NOTES}

1. KUHN T.S.. La structure des révolutions scientifiques, (1962), Paris, Flammarion, tr. fr., 1982.

2. MORIN E, Introduction à la pensée complexe, Paris, E.S.F., 1990, p. 147.

3. BERTHELOT J.-M., L'intelligence du social, Le pluralisme explicatif en sociologie, P.U.F., 1990.

4. ANSART P.. Les sociologies contemporaines, Paris, Éd. du Seuil, 1990. p. 21

5. BROHM J.-M., Les meutes sportives, critique de la domination, Paris, L'Harmattan, 1993, p. 235

6. ibid., p. 57.

7. ibid., p. 23

8. ibid., p. 68

9. ibid., p. 108.

10. GRAMSCI A., Oeuvres choisies, Paris, Éd. Sociales, 1959.

11. ALTHUSSER L., «Idéologie et Appareils Idéologiques d'État, Notes pour une recherche », La Pensée, 151, juin 1970, p. 15.

12. BROHM J.-M., Les meutes sportives, critique de la domination, op. cit., p. $268 \mathrm{sq}$

13. ibid., p. 269.

14. ABENSOUR M., «Les penseurs de l'exil », Entretien d'Edmond El Maleh, Le Monde. 2/3/1980, reproduit in Entretiens avec Le Monde. 1-Philosophies, Paris, Éd. La Découverte/Le Seuil, 1984, p. 209-219.

15. BROHM J.-M., "Le corps dans les institutions, les institutions du corps, le corps comme institution », Quel Corps ? 28/29. décembre 1985, p. 5-8. 
16. BROHM J.-M., «L'emprise au corps », ibid., p. 37

17. BROHM J.-M., "Les discours idéologiques du sport: État d'une recherche », Les Sciences de l'Éducation

pour l'ère nouvelle, 1-2, 1990, p. 134-135.

18. BROHM J.-M., «Critique des fondements de l'Éducation Physique et Sportive, Les STAPS, une imposture

majeure ", Traité critique d'Éducation Physique et Sportive, op. cit., p. 27.

19. ibid.. p. 27.

20. ibid., p. 36. cf. aussi « Une pratique pédagogique : ce que je fais concrètement », Traité critique d'Éducation Physique et Sportive, op. cit., p. 216-226 et « Pour un changement radical en Éducation Physique et Sportive ", Traité critique d'Éducation Physique et sportive, op. cit., p. 262-273.

21. BERTHELOTJ.-M., L'Intelligence du social, le pluralisme explicatif en sociologie, op. cit.,p. 50-55. LeviStrauss G, « Analyse structurale en linguistique et en anthropologie », Anthropologie structurale. Paris, Éd. Plon. 1958.

22. BOURDIEU P.. Choses dites. Paris, Éd. de Minuit. 1987, p. 24.

23. BOURDIEU P., "Champ intellectuel et projet créateur", Les Temps Modernes, $22^{\mathrm{e}}$ année, novembre, 242, 1966. p. 865-906.

24. ibid., p. 865. Ces idées seront approfondies dans « Le marché des biens symboliques ", L'année sociologique, vol. 22, 1971, p. 49- 126.

25. ibid.. p. 866, souligné par l'auteur et p. 873.

26. BOURDIEU P., «Pratiques sportives et pratiques sociales ", Congrès de l'HISPA, 1978, p. 18-19, reproduit in Questions de sociologie. Paris, Éd. de Minuit, 1980.

27. BOURDIEU P., PASSERON J.-C., Les Héritiers. Les étudiants et la culture, Paris, Éd. de Minuit, 1964. BOURDIEU P., PASSERON J.-C. La Reproduction. Éléments pour une théorie du système d'enseignement, Paris, Éd. de Minuit, 1970. BOURDIEU P., La noblesse d'État, grandes écoles et esprit de corps. Paris, Éd. de Minuit, 1989.

28. BOURDIEU P., Esquisse d'une théorie de la pratique, Genève, Droz, 1972, p. 196-197.

29. BOURDIEU P. avec WACQUANT L.J.D., Réponses, pour une anthropologie réflexive. Paris. Éd. du Seuil. 1992. p. 78. p. 84 et p. 84.

30. MORIN E., «Peut-on concevoir une science de l'autonomie?», Science avec conscience. Paris, Fayard. 1982., p. 190-202.

31. MORIN E., Introduction à la pensée complexe, Paris, op. cit.,p. 100

32. La société, par exemple, est produite par les interactions entre les individus qui la constituent. La société elle-même, comme le tout organisé et organisateur, rétroagit pour produire les individus par l'éducation, le langage, l'école. Ainsi, les individus clans leurs interactions, produisent la société, iaquelle produit les individus qui la produisent », Ibid., p. 116.

33. CHARLOT B., "L'innovation n'est plus ce qu'elle était», Autrement. ("Ainsi change l'école, l'éternel chantier des novateurs »). 136. mars 1993. p. 20-27.

34. MORIN E., «Le retour de l'événement », in Science avec conscience, op. cit., p. 135.

35. ANSART P., "Toute connaissance du social est-elle idéologique?», in Sociologie de la connaissance, (Textes réunis par Jean DUVIGNAUD), Paris, Payot, 1979, p. 41-42.

36. BOURDIEU P., Réponses, op. cit.. p. 78.

37. BOURDIEU P., «Tout est social ». Magazine Littéraire, ${ }^{\circ} 303$, octobre 1992, p. 104-110. Propos tenu après sa nomination au Collège de France. 


\section{RÉSUMÉS}

Une réflexion fondée sur les trois grands paradigmes sociologiques qui servent ou ont servi à analyser aussi bien le phénomène sportif que les pratiques corporelles en général, permet de clarifier leur fonction et leur rôle dans le champ de l'éducation physique. Trois images métaphoriques se dégagent. La réception de chacune de ces images, n'est pas identique. Si la théorie de Jean-Marie Brohm fondée sur l'idée d'Appareil Idéologique d'État est plutôt mal reçue, celle de Pierre Bourdieu pourtant également politique est assez bien acceptée. Une troisième forme de théorisation, systémique, qui 'appuie sur les positions d'Edgar Morin ne semble pas pour l'instant susciter de réactions vives. Peut-être pourrait-elle être un outil de dépassement des deux autres.

A reflection, grounded in the three main sociological paradigms which are used or have been used to analyze the phenomenon of sports as well as physical activities in general, making it possible to clarify their function and their role in the field of physical education. Through this reflection, three images (metaphorically speaking), come across. How each of these is received is not identical. While Jean-Marie Brohm's theoretical framework based on the concept of state ideological structure is rather poorly received, Pierre Bourdieu's though it is equally political is rather well accepted. A third theoretical framework, a systemic one, which is based on Edgar Morin's positions does not 'Or the time being seem to arouse any strong reactions. Perhaps it could become a tool to surpass the other two.

INDEX

Mots-clés : éducation physique, images métaphoriques, Paradigmes sociologiques, réception/ acceptation, sport

Keywords : Metaphorical images, Physical education, Reception/Acceptance, Sociological paradigms, Sports

\section{AUTEUR}

\section{YVES LE POGAM}

Maître de conférences, UFR S.T.A.P.S., université de Montpellier I, laboratoire « Corps et Culture ", enseignant CAPEPS, IUFM de Montpellier 